\title{
A simple scoring system in COVID-19 patients with neurological manifestations
}

\author{
${ }^{1}$ Hatice Yuksel, ${ }^{1}$ Ebru Bilge Dirik, ${ }^{1}$ Gorkem Tutal Gursoy, ${ }^{1}$ Ozlem Ozturk Tan, ${ }^{1}$ Hesna \\ Bektaş, ${ }^{2}$ Levent Yamanel, ${ }^{3}$ Rahmet Güner
}

${ }^{1}$ Department of Neurology, Ankara City Hospital, Ankara; ${ }^{2}$ Department of Intensive Care Unit, Gulhane Medical Faculty, Ankara; ${ }^{3}$ Department of Infectious Diseases and Clinical Microbiology, Ankara City Hospital, Ankara, Turkey

\begin{abstract}
Background: Coronavirus disease 2019 (COVID-19) has affected a rapidly growing patient population worldwide. To effectively manage the disease, physicians need tests or methods that classify patients according to their risks. Our aim is to determine the importance of mean platelet volume (MPV), neutrophil-lymphocyte ratio (NLR), MPVNLR score (MPV $\times$ Neutrophil/lymphocyte) in predicting the clinical course and mortality in hospitalized COVID-19 patients with neurological manifestations. Methods: A total of 300 patients were enrolled in the study. MPV, NLR, MPVNLR score, CRP, D-dimer, IL-6, and procalcitonin tests were obtained for each patient. Results: MPV, NLR, MPVNLR score, IL-6, D-dimer, procalcitonin, and CRP in the non-survivor group were higher than the survivor group ( $\mathrm{p}=0.001, \mathrm{p}: 0.001, \mathrm{p}=0.001, \mathrm{p}=0.001, \mathrm{p}=0.001, \mathrm{p}=0.027$, and $\mathrm{p}=0.001$, respectively). MPV, NLR, MPVNLR score, IL-6, D-dimer, procalcitonin and CRP were higher in patients with altered mental status $(\mathrm{p}=0.001, \mathrm{p}=0.001, \mathrm{p}=0.001, \mathrm{p}=0.014, \mathrm{p}=0.002, \mathrm{p}=0.026$, and $\mathrm{p}=0.001$, respectively). Conclusion: High MPV, NLR, and MPVNLR scores may be simple markers to predict mortality and mental deterioration in COVID-19.
\end{abstract}

Keywords: Coronavirus infections, mean platelet volume, mortality, neurological manifestations.

\section{INTRODUCTION}

The coronavirus disease 2019 (COVID-19) has turned into a major health problem affecting the whole world in less than a year. While it was thought that COVID-19 affected only the respiratory tract in the early periods, the multisystem nature of the disease was obvious in a short time. Available data show that most COVID-19 patients have a mild clinical presentation, but some have a severe and lifethreatening clinical course. ${ }^{1,2}$

The COVID-19 pandemic has significant mortality rate and the need for intensive care worldwide. However, the risk factors associated with these bad outcomes of COVID-19 have remained poorly understood. Currently, an important issue facing the physicians has been how to identify patients who need intensive care and who also have a high risk of morbidity and mortality. 3,4

There were recent studies that reported that simple blood tests can predict critically ill patients in COVID-19. They have investigated the various parameters such as platelet count, neutrophillymphocyte ratio (NLR), basophil-lymphocyte ratio (BLR) and their formulations as factors in determining the severity and mortality risk of COVID-19..$^{5-8}$ Mean platelet volume (MPV) has been used as an important marker in defining the severity of inflammation. ${ }^{9}$ A few studies have evaluated the role of MPV in predicting thrombosis, disease severity, and mortality in COVID-19. ${ }^{7,10}$ However, up to now, the effect of these tests has not been evaluated in patients with neurological manifestations.

Thus, in this study, we aimed firstly to determine the importance of MPV, NLR, and MPVNLR score (MPV $\times$ Neutrophil/lymphocyte) in predicting the clinical course and mortality in hospitalized COVID-19 patients with neurological manifestations; secondly, the predictive efficacy of acute inflammatory markers, including C-reactive protein (CRP), procalcitonin, D-dimer, and interleukin-6 (IL-6).

Address correspondence to: Hatice Yuksel, Ankara City Hospital, Department of Neurology, Üniversiteler Mahallesi 1604. Cadde No: 9, Çankaya/Ankara, Turkey. Tel: +90 (312) 2232263, e-mail: haticeyuksel73@yahoo.com

Date of Submission: 20 May 2021; Date of Acceptance 2 July 2021

https://doi.org/10.54029/2021rrf 


\section{METHODS}

The study population consisted of all COVID-19 patients with neurological manifestations hospitalized in Ankara City Hospital between April 2020 and September 2020.

All neurological findings were collected, including headaches, dizziness, paresthesia, paresis, speech disorder, seizure attack, and consciousness level.

If the patient's level of consciousness and interaction with the environment was normal, this was defined as normal mental status (NMS). If somnolence, agitation, delirium, confusion, and coma were present in the neurological examination, this condition was described as altered mental status (AMS).

Complete blood cell count (CBC), creatinine, CRP, D-dimer, IL-6, and procalcitonin tests were obtained for each patient on the first day of admission to the hospital.

We calculated the MPVNLR score as MPV $\times$ Neutrophil / lymphocyte.

The study was approved by the local ethical committee (Ankara City Hospital Ethics Committee).

All statistical analyses were done using IBM SPSS statistic 22.0 (Chicago, IL, USA). Data were expressed as mean \pm SD. Categorical variables were compared using the Chi-Square test. Continuous variables were compared using the Student's t-test. Receiver operating characteristic (ROC) curve analysis was used to identify optimal cut-off values of MPVNLR and other inflammation markers to identify with maximum sensitivity and specificity the prediction of mental deterioration and mortality rate. Binary logistic regression analysis was performed to detect independent factors associated with AMS and mortality. A p-value $<0.05$ was considered statistically significant.

\section{RESULTS}

A total of 300 patients, 121 (40.3\%) female, and $179(59.7 \%)$ male, were enrolled in the study. The mean age was $68.20 \pm 18.31$ years for females and $67.63 \pm 13.76$ for males.

One hundred eighty-nine patients $(63 \%)$ were treated in the intensive care unit, and 55 patients $(18.3 \%)$ needed intubation.

Ninety-seven patients died during hospital follow-up (mortality rate of $32.3 \%$ ). The platelet count was similar in both survivor and nonsurvivor groups ( $\mathrm{p}=0.785$ ). MPV, NLR, MPVNLR score, IL-6, D-dimer, procalcitonin, and CRP levels in the non-survivor group were higher than the survivor group $(\mathrm{p}=0.001, \mathrm{p}=0.001, \mathrm{p}=0.001$, $\mathrm{p}=0.001, \mathrm{p}=0.001, \mathrm{p}=0.027$, and $\mathrm{p}=0.001$, respectively)(Table $1 \mathrm{~A})$. In binary logistic regression analysis, MPV, NLR, CRP, D-dimer, IL-6, and age were found to be independent risk factors for mortality (Table1B).

MPVNLR, CRP, D-dimer, procalcitonin, IL-6 and platelet had $0.727,0.670,0.720,0.725,0.680$ and 0.495 , respectively the area under the ROC curve (AUROC) for mortality. MPVNLR cut-off point of 75.360 for mortality had a sensitivity of 79.4 , specificity of 58.9 , positive predictive value (PPV) of $48.1 \%$ and negative predictive value (NPV) of $85.6 \%$ (Table 2) (Figure1).

The most common neurological manifestation was AMS (209 patients, 69.7\%). The platelet count, hemoglobin, and creatinine levels were comparable in both AMS and NMS groups $(\mathrm{p}=0.192, \mathrm{p}=0.155$, and $\mathrm{p}=0.170$, respectively $)$. MPV, NLR, MPVNLR score, IL-6, D-dimer, procalcitonin, and CRP levels in the AMS patients were higher than the NMS patients $(\mathrm{p}=0.001$, $\mathrm{p}=0.001, \mathrm{p}=0.001, \mathrm{p}=0.014, \mathrm{p}=0.002, \mathrm{p}=0.026$ and $\mathrm{p}=0.001$, respectively) (Table $1 \mathrm{~A}$ ). In binary logistic regression analysis, MPV, NLR, and age were found to be independent risk factors for AMS (Table1B).

MPVNLR, CRP, D-dimer, procalcitonin, IL-6 and platelet count had 0.694, 0.678, 0.677, 0.709, 0.595 and 0.477 , respectively AUROC for AMS. MPVNLR cut-off point of 62.990 for AMS had a sensitivity of 63.9 , specificity of 70.3, PPV of $83.1 \%$ and NPV of $46.1 \%$ (Table 2) (Figure1).

In patients with cerebral infarction, platelet count, MPV, NLR and MPVNLR values were comparable to patients without cerebral infarction $(\mathrm{p}=0.463, \mathrm{p}=0.444, \mathrm{p}=0.861$ and $\mathrm{p}=0.941$, respectively) (Table 3 ).

Platelet count was lower in patients with seizures than in patients without seizures (p: 0.015). MPV, NLR, and MPVNLR values were similar in both groups $(\mathrm{p}=0.441, \mathrm{p}=0.077$ and $\mathrm{p}=0.121$, respectively) (Table 3).

The NLR was significantly lower in patients with headache $(\mathrm{p}=0.031)$, but the platelet count was similar to those without headache ( $\mathrm{p}=0.447)$. Although MPV and MPVNLR values in patients with headache were lower, and the difference was not significant ( $\mathrm{p}=0.053$ and $\mathrm{p}=0.065)$ (Table 3 ).

\section{DISCUSSION}

Our study showed that the mortality rate is high in COVID-19 patients with neurological 
Table 1A: Comparison of demographic and laboratory of the NMS group versus AMS group and survivor group versus non-survivor group. B. Independent factors associated with mortality and altered mental status in binary logistic regression analysis

\begin{tabular}{|c|c|c|c|c|c|c|c|c|c|c|}
\hline \multicolumn{11}{|l|}{ A } \\
\hline & \multicolumn{2}{|l|}{$\begin{array}{l}\text { Survivor } \\
\text { n: } 203\end{array}$} & \multicolumn{2}{|c|}{$\begin{array}{l}\text { Non-survivor } \\
\text { n: } 97\end{array}$} & \multicolumn{3}{|c|}{$\begin{array}{l}\text { NMS } \\
\text { n: } 91\end{array}$} & \multicolumn{2}{|c|}{$\begin{array}{c}\text { AMS } \\
\text { n: } 209\end{array}$} & $\mathbf{p}$ \\
\hline Age & \multicolumn{2}{|c|}{$65.67 \pm 16.66$} & \multicolumn{2}{|c|}{$72.43 \pm 12.44$} & 0.001 & \multicolumn{2}{|c|}{$61.93 \pm 17.17$} & \multicolumn{2}{|c|}{$70.44 \pm 14.34$} & 0.001 \\
\hline Sex Female / Male & \multicolumn{2}{|l|}{$121 / 82$} & \multicolumn{2}{|c|}{$58 / 39$} & 0.975 & \multicolumn{2}{|c|}{$33 / 58$} & \multicolumn{2}{|c|}{$88 / 121$} & 0.343 \\
\hline Creatinine $\mathrm{mg} / \mathrm{dL}$ & \multicolumn{2}{|c|}{$1.13 \pm 1.16$} & \multicolumn{2}{|c|}{$1.54 \pm 1.44$} & 0.015 & \multicolumn{2}{|c|}{$1.11 \pm 1.43$} & \multicolumn{2}{|c|}{$1.33 \pm 1.19$} & 0.170 \\
\hline Hemoglobin g/dL & \multicolumn{2}{|c|}{$12.28 \pm 2.28$} & \multicolumn{2}{|c|}{$11.55 \pm 2.31$} & 0.012 & \multicolumn{2}{|c|}{$12.33 \pm 2.17$} & \multicolumn{2}{|c|}{$11.92 \pm 2.37$} & 0.155 \\
\hline Neutrophil $10^{9} / \mathrm{L}$ & \multicolumn{2}{|c|}{$6.40 \pm 3.60$} & \multicolumn{2}{|c|}{$11.37 \pm 9.22$} & 0.001 & \multicolumn{2}{|c|}{$5.84 \pm 3.03$} & \multicolumn{2}{|c|}{$8.95 \pm 7.26$} & 0.001 \\
\hline Lymphocyte $10^{9} / \mathrm{L}$ & \multicolumn{2}{|c|}{$1.21 \pm 1.28$} & \multicolumn{2}{|c|}{$0.90 \pm .64$} & 0.025 & \multicolumn{2}{|c|}{$1.41 \pm 1.75$} & \multicolumn{2}{|c|}{$0.98 \pm 0.65$} & 0.002 \\
\hline Platelets $10^{9} / \mathrm{L}$ & \multicolumn{2}{|c|}{$262.86 \pm 117.64$} & 258 & 152.58 & 0.785 & $276.26 \pm$ & 24.30 & 254.9 & 131.78 & 0.192 \\
\hline MPV fL & $8.74 \pm 1.14$ & & 9.46 & & 0.001 & $8.57 \pm$ & & 9.1 & 1.38 & 0.001 \\
\hline CRP mg/dL & $61.40 \pm 62$ & & 112 & 81.53 & 0.001 & $51.98 \pm$ & 5.26 & 89.1 & 73.77 & 0.001 \\
\hline D-dimer mg/L & $2.90 \pm 5.81$ & & 7.65 & & 0.001 & $2.37 \pm$ & & & 8.30 & 0.002 \\
\hline Procalcitonin $\mu / \mathrm{L}$ & $0.85 \pm 3.85$ & & 3.36 & & 0.027 & $0.42 \pm$ & & 2.1 & 10.92 & 0.026 \\
\hline IL-6 $\mathrm{pg} / \mathrm{mL}$ & $45.95 \pm 73$ & & 169 & 268.65 & 0.001 & $53.13 \pm$ & 9.90 & 101. & 197.91 & 0.014 \\
\hline NLR & $7.93 \pm 7.28$ & & & & 0.001 & 6.4 & & 14 & 23.50 & 0.001 \\
\hline MPVNLR score & $69.09 \pm 61$ & & 213 & 00.37 & 0.001 & $55.01 \pm$ & 8.96 & 142 & 285.03 & 0.001 \\
\hline B & & & & & & & & & & \\
\hline & & Mor & lity & & & & ared $N$ & Mental & us & \\
\hline & $\mathbf{p}$ & & & $\%$ & & $\mathbf{p}$ & $\operatorname{Exp}$ & $(\mathrm{B})$ & $\% 95$ & CI \\
\hline Age & 0.017 & & & 1.006 & 061 & 0.001 & 1.0 & 042 & 1.019 & 1.065 \\
\hline Platelets $10^{9} / \mathrm{L}$ & 0.139 & & & 0.999 & 005 & 0.598 & 1.0 & 001 & $0.998-$ & 1.004 \\
\hline MPV fL & 0.031 & & & 1.028 & 756 & 0.046 & 1.4 & 430 & $1.006-$ & 2.033 \\
\hline CRP $\mathrm{mg} / \mathrm{dL}$ & 0.006 & & & 1.002 & .012 & 0.223 & 1.0 & 004 & $0.998-$ & 1.010 \\
\hline D-dimer mg/L & 0.001 & & & $1.042-$ & .161 & 0.175 & 1.0 & 050 & $0.978-$ & -1.127 \\
\hline Procalcitonin $\mu / \mathrm{L}$ & 0.358 & & & $0.940-$ & .023 & 0.190 & 1.3 & 384 & $0.851-$ & 2.251 \\
\hline Interleukin- $6 \mathrm{pg} / \mathrm{mL}$ & 0.003 & & & $1.001-$ & .007 & 0.856 & 1.0 & 000 & $0.998-$ & -1.003 \\
\hline NLR & 0.001 & & & $1.033-$ & .107 & 0.025 & 1.0 & 061 & $1.007-$ & -1.117 \\
\hline
\end{tabular}

NMS: Normal mental status, AMS: Altered mental status, MPV: Mean platelet volume, CRP: C reactive protein, IL-6: Interleukin-6, NLR: Neutrophil Lymphocyte ratio, MPVNLR score: MPV×Neutrophil/lymphocyte

manifestations. MPVNLR score calculated from simple complete blood count parameters was successful in predicting mortality. Also, the MPVNLR score was efficient in detecting mental deterioration. Although procalcitonin had a predictive power comparable to MPVNLR score in detecting mental deterioration, only MPV, NLR, and age were found to be independent risk factors in predicting mental deterioration.

Some studies reported that the neurological involvement of COVID-19 patients was associated with the severity of the disease, and could indicate high mortality. ${ }^{11,12}$ Our study found that the mortality rate was $32.3 \%$ in hospitalized patients with neurological manifestations. All available data revealed that the healthcare system had to have tests and scoring systems that can quickly identify patients at risk that can be applied easily.

In many studies, NLR, BLR, platelet count and platelet-to-lymphocyte ratio have been used as markers to determine the disease's severity and mortality rate in COVID-19.5,6,13 MPV has been evaluated in a few studies. ${ }^{7,14}$ In a study, it was reported that the high values of MPR score obtained by proportioning MPV to the platelet count predicted the development of 
Table 2: AUC, sensitivity, specificity, positive and negative predictive values to predict the mortality and altered mental status

\begin{tabular}{|c|c|c|c|c|c|c|}
\hline & Cut-off & AUROC & Sensitivity $\%$ & Specificity $\%$ & PPV \% & NPV\% \\
\hline \multicolumn{7}{|l|}{ Mortality } \\
\hline MPVNLR score & 75.360 & 0.727 & 79.4 & 58.9 & 48.1 & 85.6 \\
\hline CRP & 63.500 & 0.670 & 73.2 & 58.1 & 45.5 & 81.9 \\
\hline D-dimer & 1.895 & 0.720 & 75.0 & 57.1 & 45.3 & 82.9 \\
\hline Procalcitonin & 0.125 & 0.725 & 76.3 & 63.1 & 49.7 & 84.8 \\
\hline Interleukin-6 & 32.900 & 0.680 & 73.2 & 51.6 & 44.8 & 78.2 \\
\hline Platelet & 245.500 & 0.495 & 48.5 & 50.2 & 31.8 & 67.1 \\
\hline \multicolumn{7}{|c|}{ Altered Mental Status } \\
\hline MPVNLR score & 62.990 & 0.694 & 63.9 & 70.3 & 83.1 & 46.1 \\
\hline CRP & 50.500 & 0.678 & 62.2 & 71.4 & 83.3 & 45.1 \\
\hline D-dimer & 1.245 & 0.677 & 61.5 & 65.9 & 80.5 & 42.9 \\
\hline Procalcitonin & 0.095 & 0.709 & 69.4 & 71.4 & 84.8 & 50.8 \\
\hline Interleukin-6 & 24.050 & 0.595 & 60.9 & 54.1 & 79.1 & 54.1 \\
\hline Platelet & 244.300 & 0.477 & 47.4 & 45.1 & 66.4 & 27.2 \\
\hline
\end{tabular}

MPVNLR score: MPV $\times$ Neutrophil/lymphocyte, CRP: C-reactive protein

severe pneumonia in COVID-19..$^{15}$ Guclu et al. reported that high MPV levels are associated with an increased risk of mortality. ${ }^{16}$ In our study, we demonstrated that MPV is an independent marker for predicting mortality in COVID-19 patients with neurological manifestations. At the same time, the ROC analysis of mortality showed that the MPVNLR score has a higher AUC than all parameters.

COVID-19 can lead to broad-spectrum neurological disorders. Neurological symptoms and signs may not be apparent at the beginning.
However, in the course of time, neurological involvement may progress, become more severe, and even life-threatening. The development of mental impairment has been associated with a high mortality rate. ${ }^{17}$ In our study, mental deterioration was the most common neurological manifestation, and the mortality rate in these patients was significantly higher (44\%). We detected high MPV levels in patients with mental deterioration. We also demonstrated that high MPV is an independent marker for mental deterioration. Metabolic alterations and hypoxia
MORTALITYY

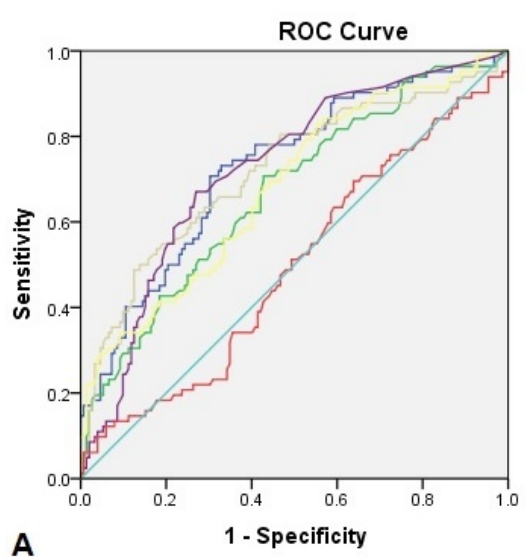

ALTERED MENTAL STATUS

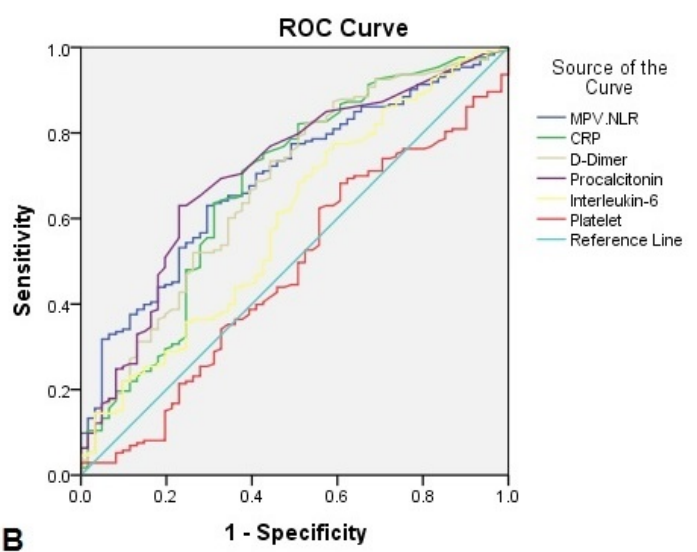

Figure 1. Receiver operator characteristic (ROC) curves of laboratory parameters for predicting mortality and altered mental status. 
Table 3: MPV, platelet count, NLR and MPVNLR score in neurological manifestations

\begin{tabular}{|c|c|c|c|c|}
\hline & MPV & Platelet & NLR & MPVNLR \\
\hline \multicolumn{5}{|l|}{ Cerebral infarct } \\
\hline Absent (n:190) & $8.90 \pm 1.29$ & $259.05 \pm 124.16$ & $12.48 \pm 21.43$ & $119.50 \pm 277.87$ \\
\hline Present (n:88) & $9.02 \pm 1.02$ & $271.38 \pm 142.26$ & $12.96 \pm 19.63$ & $117.08 \pm 180.36$ \\
\hline $\mathrm{p}$ & 0.444 & 0.463 & 0.861 & 0.941 \\
\hline \multicolumn{5}{|l|}{ Seizure } \\
\hline Absent (n:255) & $8.95 \pm 1.30$ & $269.07 \pm 128.72$ & $10.66 \pm 13.84$ & $96.65 \pm 129.29$ \\
\hline Present (n:45) & $9.11 \pm 1.29$ & $218.20 \pm 128.25$ & $21.43 \pm 39.52$ & $223.80 \pm 536.60$ \\
\hline $\mathrm{p}$ & 0.441 & 0.015 & 0.077 & 0.121 \\
\hline \multicolumn{5}{|l|}{ Headache } \\
\hline Absent (n:260) & $9.03 \pm 1.34$ & $259.20 \pm 131.15$ & $13.27 \pm 21.46$ & $125.96=$ \\
\hline Present (n:40) & $8.60 \pm 0.89$ & $276.00 \pm 120.58$ & $5.86 \pm 4.85$ & $49.89 \pm 40.70$ \\
\hline $\mathrm{p}$ & 0.053 & 0.447 & 0.031 & 0.065 \\
\hline
\end{tabular}

MPV: Mean platelet volume, NLR: Neutrophil Lymphocyte ratio,

MPVNLR score: MPV $\times$ Neutrophil/lymphocyte

may be responsible for mental changes in these patients. Microangiopathic thrombi, which cannot be detected by neuroimaging tests, may be another factor causing mental deterioration. Autopsy studies showed thrombotic microangiopathy and intravascular platelet aggregates in lung, kidney, liver, and brain. ${ }^{14,18}$

COVID-19 reduces the activity of the angiotensin receptor (ACE) 2 and increases angiotensin II levels. Angiotensin II, a potent vasoconstrictor, activates the coagulation cascade by increased platelet activation and size..$^{14,19}$ COVID-19 causes a decrease in the platelet count through many mechanisms. As a hemostatic response to this decrease in platelets, thrombocyte production increases in the body, and in this case, a large number of young platelets enter the circulation. Young platelets are larger in size and more active. Therefore, platelets activated by SARS-CoV-2 infection may be responsible for the microangiopathic thrombosis observed in biopsy specimens of COVID-19 patients..$^{14,19,20}$ The increased MPV level in COVID-19 may predict thrombotic microangiopathy in many organs, including the brain, lung, kidney, and heart. In patients with high MPV, initiation of an effective anticoagulant and anti-platelet therapy may prevent damage in these organs. ${ }^{14,20}$

In our study, MPV and platelet counts in patients with ischemic stroke detected on neuroimaging were not different from those without ischemic stroke. Consistent with our study, Melazzini et al. did not detect an increase in thrombocyte count in patients with venous thrombosis detected by imaging methods. ${ }^{21}$

Another neurological manifestation evaluated in our study was epileptic seizures. We found a significant decrease in platelet count in patients with seizures, but we could not detect a substantial increase in MPV. We could not find any study on this subject in the literature.

Trigo et al. showed that headache was associated with a low mortality rate and good prognosis. The headache had an inverse relationship with abnormal platelet count, but MPV was not evaluated in their study. ${ }^{22}$ We did not find a significant relationship between headache and MPV or platelet count.

In conclusion, our study is the first study investigating the effect of complete blood count parameters such as MPV, platelet count, and NLR on mortality rate and clinical course in COVID-19 patients with neurological manifestations. High MPV, NLR, and MPVNLR scores are simple and useful markers for predicting mortality and mental impairment. Our results support that patients with high values in these parameters, especially those accompanied by significant neurological findings, should be managed in the intensive care setting.

\section{DISCLOSURE}

Financial support: None

Conflict of interest: None

\section{REFERENCES}

1. Patel U, Mehta D, Shah D, et al. Early epidemiological indicators, outcomes, and interventions of COVID-19 pandemic: A systematic review. J Glob Health 2020; 10(2): 020506.

2. Wang Z, Deng H, Ou C, et al. Clinical symptoms, comorbidities and complications in severe and 
non-severe patients with COVID-19: A systematic review and meta-analysis without cases duplication. Medicine 2020; 99: e23327.

3. Bertsimas D, Lukin G, Mingardi L, et al. COVID-19 mortality risk assessment: An international multicenter study. PLOS ONE 2020; 15: e0243262.

4. Chen R, Liang W, Jiang M, et al. Risk factors of fatal outcome in hospitalized subjects with coronavirus disease 2019 from a nationwide analysis in China. Chest 2020; 158: 97-105.

5. Lin S, Mao W, Zou Q, Lu S, Zheng S. Associations between hematological parameters and disease severity in patients with SARS-CoV-2 infection. $J$ Clin Lab Anal 2021;35(1):223604.

6. Liu Y, Sun W, Guo Y, et al. Association between platelet parameters and mortality in coronavirus disease 2019: Retrospective cohort study. Platelets 2020; 31: 490-6.

7. Pan $\mathrm{Y}, \mathrm{Ye} \mathrm{G}, \mathrm{Zeng} \mathrm{X}$, et al. Can routine laboratory tests discriminate SARS-CoV-2-infected pneumonia from other causes of community-acquired pneumonia? Clin Transl Med 2020; 10: 161-8.

8. Usul E, Şan İ, Bekgöz B, Şahin A. Role of hematological parameters in COVID-19 patients in the emergency room. Biomark Med 2020; 14(13): 1207-15.

9. Talukdar M, Barui G, Adhikari A, Karmakar R, Ghosh UC, Das TK. A study on association between common haematological parameters and disease activity in rheumatoid arthritis. J Clin Diagn Res 2017; 11(1): EC01-EC04.

10. Barrett TJ, Lee AH, Xia Y, et al. Platelet and vascular biomarkers associate with thrombosis and death in coronavirus disease. Circ Res 2020; 127: 945-7.

11. Mao L, Jin $\mathrm{H}$, Wang $\mathrm{M}$, et al. Neurologic manifestations of hospitalized patients with coronavirus disease 2019 in Wuhan, China. JAMA Neurol 2020; 77: 683.

12. Yuksel H, Gursoy GT, Dirik EB, et al. Neurological manifestations of COVID-19 in confirmed and probable cases: A descriptive study from a large tertiary care center. J Clin Neurosci 2021; 86: 97-102.

13. Liang J, Nong S, Jiang L, et al. Correlations of disease severity and age with hematology parameter variations in patients with COVID-19 pre- and posttreatment. J Clin Lab Anal 2021;35(1):e23609.

14. Wool GD, Miller JL. The Impact of COVID-19 disease on platelets and coagulation. Pathobiology 2021;88(1):15-27.

15. Zhong Q, Peng J. Mean platelet volume/platelet count ratio predicts severe pneumonia of COVID-19. JClin Lab Anal 2021;35(1):e23607.

16. Güçlü E, Kocayiğit H, Okan HD, et al. Effect of COVID-19 on platelet count and its indices. Rev Assoc Med Bras 2020; 66: 1122-7.

17. Tyson B, Erdodi L, Ray S, Agarwal P. Altered mental status in 71 deaths due to COVID-19. Int J Neurosci 2020 Oct $1 ; 1-4$.

18. Jensen MP, Le Quesne J, Officer-Jones L, C et al. Neuropathological findings in two patients with fatal COVID-19. Neuropathol Appl Neurobiol 2020; nan. 12662.

19. Taha M, Sano D, Hanoudi S, et al. Platelets and renal failure in the SARS-CoV-2 syndrome. Platelets 2020; 1-8.

20. Zhang S, Liu Y, Wang X, et al. SARS-CoV-2 binds platelet ACE2 to enhance thrombosis in COVID-19. J Hematol Oncol 2020; 13: 120.

21. Melazzini F, Colaneri M, Fumoso F, et al. Venous thromboembolism and COVID-19: a single center experience from an academic tertiary referral hospital of Northern Italy. Intern Emerg Med 2021;16(5):114152.

22. Trigo J, García-Azorín D, Planchuelo-Gómez Á, et al. Factors associated with the presence of headache in hospitalized COVID-19 patients and impact on prognosis: a retrospective cohort study. $J$ Headache Pain 2020; 21: 94. 